\title{
Editorial
}

\section{Advances in Rehabilitation and Assistive Robots for Restoring Limb Function in Persons with Movement Disorders}

\author{
Fan Gao, ${ }^{1}$ Guanglin $\mathrm{Li}^{2}{ }^{2}$ Huapeng Wu, ${ }^{3}$ Qining Wang, ${ }^{4} \mathrm{Jie} \mathrm{Liu}^{5}$ and Justin Keogh ${ }^{6}$ \\ ${ }^{1}$ Department of Health Care Sciences, UT Southwestern Medical Center at Dallas, Dallas, TX 75390, USA \\ ${ }^{2}$ Research Center for Neural Engineering, The Institute of Biomedical and Health Engineering, \\ Shenzhen Institutes of Advanced Technology, Shenzhen 518055, China \\ ${ }^{3}$ School of Energy Systems, Lappeenranta University of Technology, 53851 Lappeenranta, Finland \\ ${ }^{4}$ The Robotics Research Group, College of Engineering, Peking University, Beijing 100871, China \\ ${ }^{5}$ The Sensory Motor Performance Program, Rehabilitation Institute of Chicago, Chicago, IL 60611, USA \\ ${ }^{6}$ Faculty of Health Sciences and Medicine, Bond University, Robina, QLD 4226, Australia \\ Correspondence should be addressed to Fan Gao; fan.gao@utsouthwestern.edu
}

Received 3 July 2016; Accepted 3 July 2016

Copyright (c) 2016 Fan Gao et al. This is an open access article distributed under the Creative Commons Attribution License, which permits unrestricted use, distribution, and reproduction in any medium, provided the original work is properly cited.

People with movement disorders are plagued with debilitating conditions, which significantly degrade their quality of life. Traditional rehabilitation typically involves intensive interaction between patients and therapists. While effective, traditional rehabilitation cannot keep abreast of the increasing patient population primarily attributed to a higher surviving rate after diseases and/or injuries. Furthermore, patients living in the rural areas have fairly limited access to rehabilitation services. In the past two decades, tremendous efforts have been put into developing rehabilitation and assistive robots to facilitate the rehabilitation training while relieving the physical involvement of therapists and/or lowering the related cost. Most notably, the rehabilitation and assistive robots have been significantly advanced with developments in actuators, sensors, microprocessors, and mobile software platforms. However, unlike traditional robotics, the intimate interaction between robot and human in rehabilitation robots indicates that the success is also closely related to a thorough understanding of the human neuromuscular aspects and human-machine interaction.

This special issue primarily aims to gather the latest achievements in rehabilitation robots, exoskeletons, and prostheses including the following topics: (a) development of rehabilitation robots, exoskeleton, and upper/lower limb prostheses driven by bionics; (b) functional evaluation of rehabilitation robots, exoskeleton, and upper/lower limb prostheses with an emphasis on human movement biomechanics; (c) musculoskeletal modeling and simulation of human movements while wearing exoskeleton or prostheses; (d) noninvasive human-machine interface based on electromyography and/or electroencephalogram; (e) sensors for monitoring kinematics/kinetics, as well as biological signals in real time; (f) innovative actuators and control algorithms applied to rehabilitation robots, exoskeletons, and prostheses.

In this special issue, collective studies address the aforementioned key elements via both technical and biomechanical approaches. A reconfigurable robotic hand exoskeleton was proposed to meet the fast growing need in hand rehabilitation. A novel control algorithm integrating sliding model control with cerebellar model articulation controller neural network was implemented in lower limb exoskeleton to enhance the coordination between patient and exoskeleton. An upper limb exoskeleton was enhanced with integrated optical cameras to offer more accurate estimation of joint posture than traditional motion capture system. A hybrid upper limb rehabilitation system consisting of a shoulderelbow-forearm exoskeleton and a robotic manipulator was validated and tested in the clinic. The characteristics of muscle-tendon stimulation such as perception threshold and vibration frequency significantly influenced the muscle forces as well as the reaction time. Patellar retention was found to be superior to patellar replacement in knee arthroplasty 
via a comprehensive computer simulation. These collective studies, as part of the latest representative work, offered some new insights into the development and implementation of rehabilitation and assistive robots.

Fan Gao

Guanglin $\mathrm{Li}$

Huapeng $W u$

Qining Wang

Jie Liu

Justin Keogh 


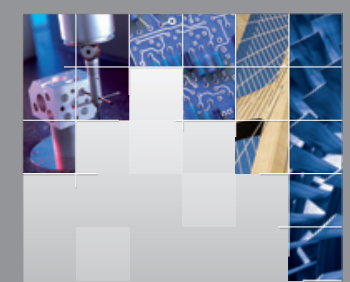

\section{Enfincering}
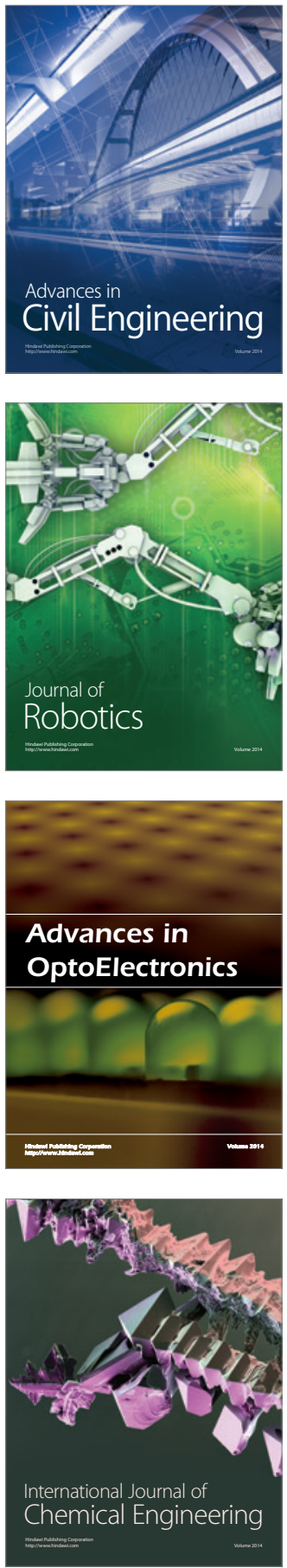

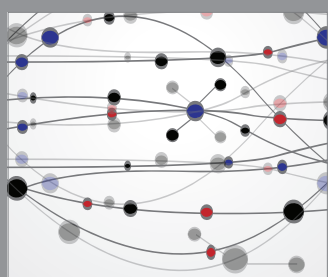

The Scientific World Journal

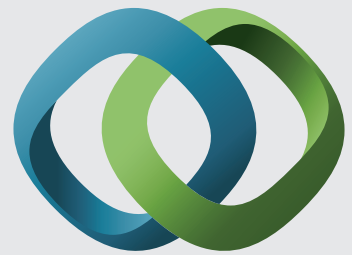

\section{Hindawi}

Submit your manuscripts at

http://www.hindawi.com
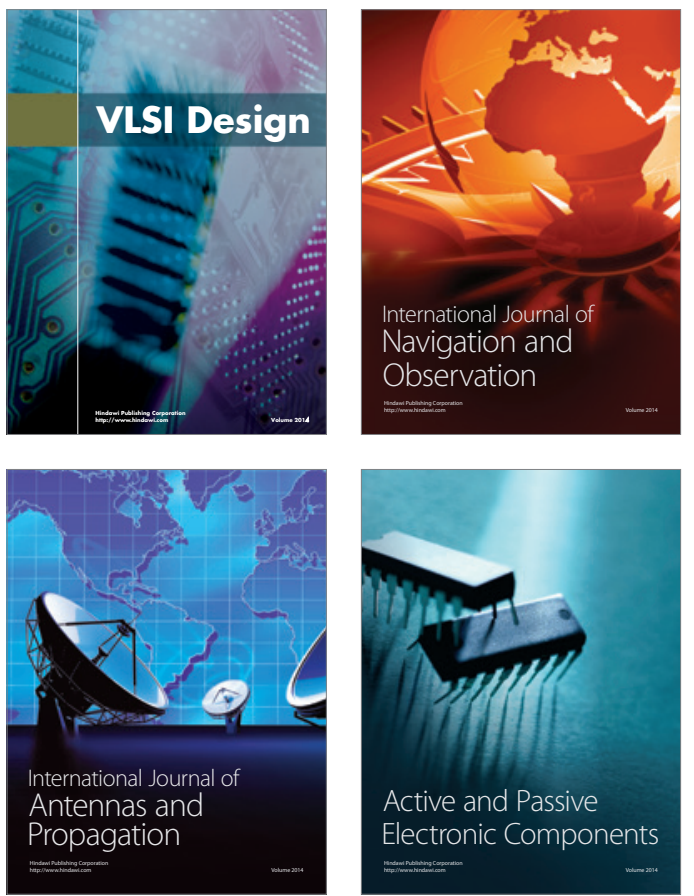
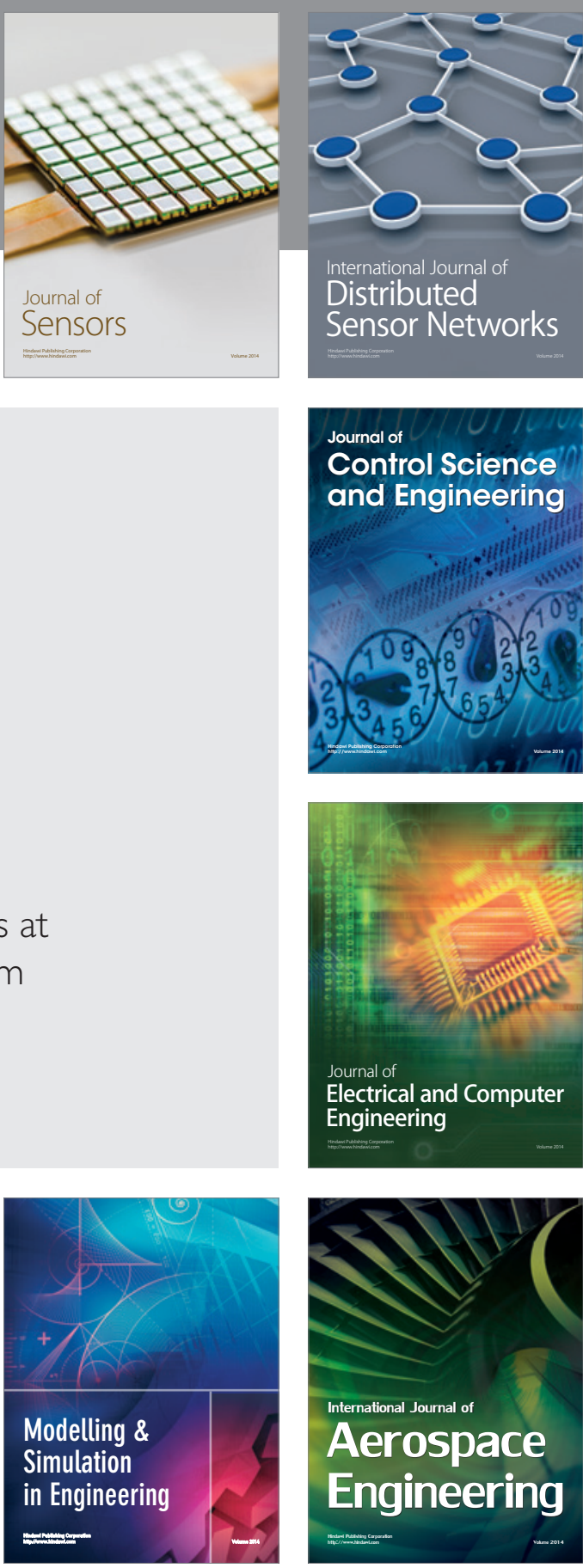

International Journal of

Distributed

Sensor Networks

Journal of

Control Science

and Engineering
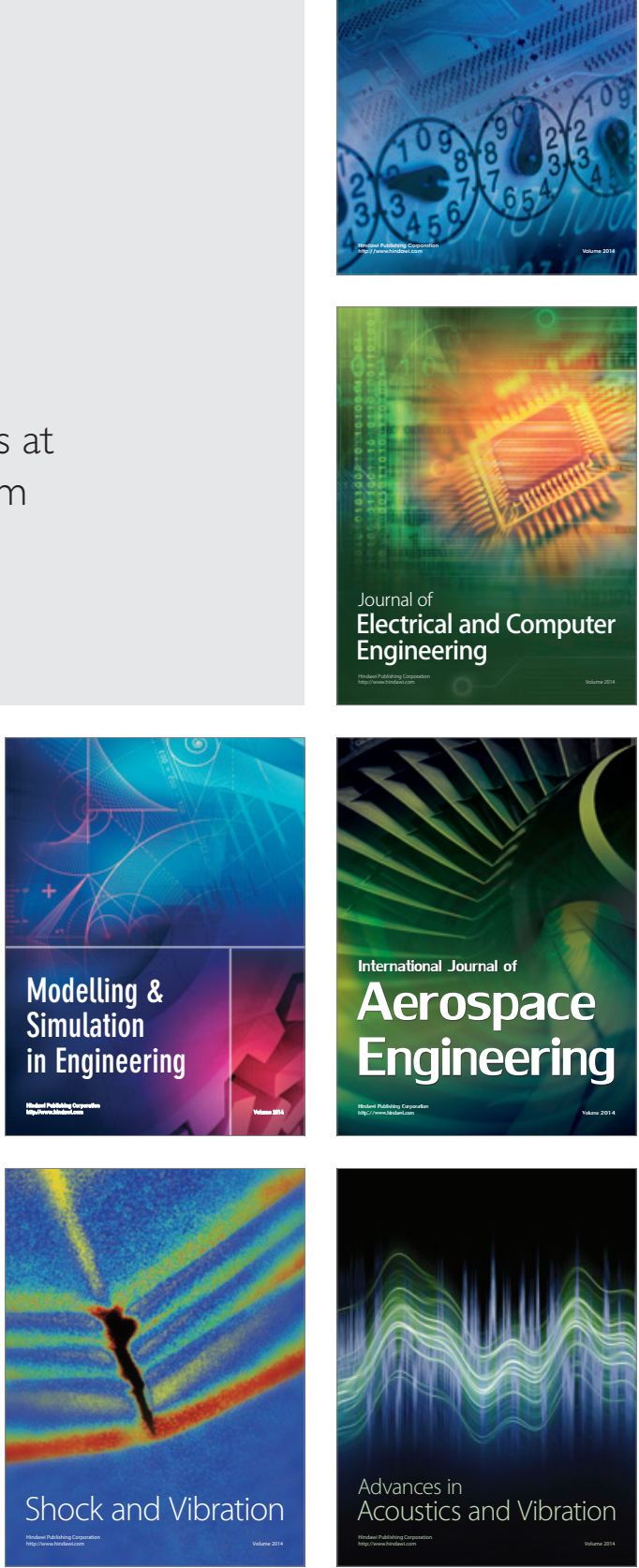